Detection of muscle gap by L-BIA in muscle injuries: clinical prognosis

This content has been downloaded from IOPscience. Please scroll down to see the full text.

2017 Physiol. Meas. 38 L1

(http://iopscience.iop.org/0967-3334/38/7/L1)

View the table of contents for this issue, or go to the journal homepage for more

Download details:

IP Address: 147.83.208.79

This content was downloaded on 22/06/2017 at 08:52

Please note that terms and conditions apply.

You may also be interested in:

Effects of muscle injury severity on localized bioimpedance measurements

L Nescolarde, J Yanguas, H Lukaski et al.

Localized bioimpedance to assess muscle injury

L Nescolarde, J Yanguas, $\mathrm{H}$ Lukaski et al.

Multi-frequency bioimpedance in equine muscle assessment

Adrian Paul Harrison, Vibeke Sødring Elbrønd, Kiwa Riis-Olesen et al.

In vivo EIS characterization of tumour tissue properties is dominated by excess extracellular fluid Christina Skourou, Andreas Rohr, P Jack Hoopes et al.

Measurement errors in multi-frequency bio-impedance analyzers

P Bogónez-Franco, L Nescolarde, $R$ Bragós et al.

$\underline{\text { Anterior thigh composition measured using ultrasound imaging to quantify relative thickness of }}$ muscle and non-contractile tissue: a potential biomarker for musculoskeletal health

Sandra Agyapong-Badu, Martin Warner, Dinesh Samuel et al.

Body composition electrical model

Fansan Zhu, Edward F Leonard and Nathan W Levin

Bioimpedance of soft tissue under compression

R E Dodde, J L Bull and A J Shih

Segmental thoracic bioimpedance measurement in CAPD

L Nescolarde, M A García-González, J Rosell-Ferrer et al. 


\title{
Detection of muscle gap by L-BIA in muscle injuries: clinical prognosis
}

\author{
L Nescolarde ${ }^{1,6}$, J Yanguas ${ }^{2}$, J Terricabras ${ }^{2,3}$, H Lukaski ${ }^{4}$, \\ X Alomar ${ }^{5}$, J Rosell-Ferrer ${ }^{1}$ and G Rodas ${ }^{2}$ \\ ${ }^{1}$ Department of Electronic Engineering, Universitat Politècnica de Catalunya, c/Jordi \\ Girona, 1-3, Edifici C4, 08034 Barcelona, Spain \\ 2 Medical Department, Futbol Club Barcelona, FIFA Medical Center of Excelence, \\ Ciutat Esportiva Futbol Club Barcelona, Av. del Sol s/n, 08790 Sant Joan Despí, \\ Barcelona, Spain \\ ${ }^{3}$ Department of Surgery, Universitat Autònoma de Barcelona, Edifici U, Campus \\ UAB, 08913-Bellaterra, Barcelona, Spain \\ ${ }^{4}$ Department of Kinesiology and Public Health Education, Hyslop Sports Center, \\ University of North Dakota, Grand Forks, ND 58202, United States of America \\ 5 Department of Radiology, Centres Mèdics Creu Blanca, Av. Reina Elisenda de \\ Montcada 15-17, 08034 Barcelona, Spain
}

E-mail: lexa.nescolarde@upc.edu

Received 30 March 2017, revised 28 April 2017

Accepted for publication 10 May 2017

Published 22 June 2017

\begin{abstract}
Sport-related muscle injury classifications are based basically on imaging criteria such as ultrasound (US) and magnetic resonance imaging (MRI) without consensus because of a lack of clinical prognostics for return-toplay (RTP), which is conditioned upon the severity of the injury, and this in turn with the muscle gap (muscular fibers retraction). Recently, Futbol Club Barcelona's medical department proposed a new muscle injury classification in which muscle gap plays an important role, with the drawback that it is not always possible to identify by MRI. Localized bioimpedance measurement (L-BIA) has emerged as a non-invasive technique for supporting US and MRI to quantify the disrupted soft tissue structure in injured muscles. Objective: To correlate the severity of the injury according to the gap with the RTP, through the percent of change in resistance $(R)$, reactance $(X c)$ and phase-angle (PA) by L-BIA measurements in 22 muscle injuries. Main results: After grouping the data according to the muscle gap (by MRI exam), there were significant differences in $R$ between grade 1 and grade $2 \mathrm{f}$ (myotendinous or myofascial muscle injury with feather-like appearance), as well as between grade $2 \mathrm{f}$ and grade $2 \mathrm{~g}$ (myotendinous or myofascial muscle injury with feather and gap).
\end{abstract}

\footnotetext{
${ }^{6}$ Author to whom any correspondence should be addressed.
} 
The $X c$ and PA values decrease significantly between each grade (i.e. 1 versus $2 \mathrm{f}, 1$ versus $2 \mathrm{~g}$ and $2 \mathrm{f}$ versus $2 \mathrm{~g}$ ). In addition, the severity of the muscle gap adversely affected the RTP with significant differences observed between 1 and $2 \mathrm{~g}$ as well as between $2 \mathrm{f}$ and $2 \mathrm{~g}$. Significance: These results show that L-BIA could aid MRI and US in identifying the severity of an injured muscle according to muscle gap and therefore to accurately predict the RTP.

Keywords: localized bioimpedance, grade of muscle injury, return-to-play, professional football players

(Some figures may appear in colour only in the online journal)

\section{Introduction}

Injuries to skeletal muscles are prevalent in competitive sports, particularly in football, with an incidence of around $30 \%$ of total injuries (Ekstrand et al 2012). Sport-related muscle injury classifications (Hamilton et al 2014) are primarily based on imaging criteria such as ultrasound (US) and more recently magnetic resonance imaging (MRI). However, the prognosis using these criteria is still not clear. There is strong evidence that a great part of the returnto-play (RTP) decision is conditioned upon the severity of the injury (Pedret et al 2015) and this is associated with muscle gap (muscular fibers retraction). Some authors (Slavotinek et al 2002, Verrall et al 2006, Connell et al 2014) have affirmed that the amount of edema of the injured muscle is associated with longer recovery times, whereas others (Schneider-Kolsky et al 2006, Reurink et al 2015) find no strong evidence of any MRI findings able to give a prognosis of RTP. Pedret et al (2015) points out that the amount of edema is relatively nonsignificant in comparison with other important factors: the extent of the muscle gap and the involvement of the surrounding tissues. Recently, a new classification of muscle injuries has been proposed (Valle et al 2016) in which muscle gap plays an important role, with the drawback that it is not always possible to identify by MRI.

Localized bioimpedance measurement (L-BIA) has emerged as an alternative, low-cost or accessible technique for supporting imaging in quantifying the disrupted soft tissue structure in injured muscles (Nescolarde et al 2015). It utilizes measurements of the passive electrical characteristics of healthy tissue in response to the administration of a low-intensity alternating current to identify differences in muscle pathology and severity of injury. Resistance $(R)$ is the opposition to the conduction of applied current and is inversely related to fluid volume, and reactance or capacitance $(X c)$ is the delay in the conduction of current on cell membranes and tissue interfaces (Lukaski 2013). In a group of professional football players, $R$ measured at the site of the injury was modified by muscle fluid accumulation (edema) whereas $X c$ was modified due to muscle cell disruption. The magnitude of the decrease in both parameters $24 \mathrm{~h}$ after injury was consistent with the severity of the injury previously diagnosed by MRI. The severity of the injury was evidenced by a greater reduction in $X c$, compared to the contralateral non-injured muscle, while the amount of edema demonstrated by a reduction in $R$ did not show a good correlation (Nescolarde et al 2013, 2015). In this way, L-BIA appears to be a useful non-invasive tool to quantify structural tissue damage independent of the amount of edema in classifying its severity and to help in the follow-up until RTP.

The aim of this work is to use L-BIA measurements to correlate the severity of an injury according to the muscle gap with the RTP. 


\section{Methods}

\subsection{Muscle injury grouped according to gap}

Twenty-two muscle injuries, 12 hamstrings; 6 rectus femoris; 3 adductor longus and 1 gastrocnemius (medial head), in 18 male professional football players (20-28 years) were evaluated taking into account the presence of the gap, supported by a 3.0 T MRI system (Magnetom VERIO, Siemens Medical Solutions, version VB 17) during a period of four seasons, ending in June 2016. The Medical Committee of Futbol Club Barcelona approved the study and each volunteer provided oral and written informed consent before participation in the study. The muscle injuries were grouped according to the presence of muscle gap identified by MRI exam $24 \mathrm{~h}$ after injury:

- Grade 1: Small area of edema, either myotendinous or myofascial $(<10 \%$ of cross sectional area (CSA) of the muscle affected and $<5 \mathrm{~cm}$ of craniocaudal length) with no gap.

- Grade 2f: Moderate amount of edema (10-50\% of CSA and 5-15 cm of length) with a feather-like image and no gap.

- Grade 2g: Moderate amount of edema (10-50\% of CSA and 5-15 cm of length) with a feather-like image and gap.

\section{2. $L-B I A$}

The L-BIA measurements at $50 \mathrm{kHz}$ (BIA-101 Anniversary; AKERN-Srl, Florence, Italy) were done $24 \mathrm{~h}$ after injury and on the day of RTP to quantify the percent of change, with respect to contralateral non-injured side, $R, X c$ and phase-angle (PA).

$R, X c$ and the PA, determined at the contralateral (non-injured side), considered as the reference value, as well as the injured side values, were both measured $24 \mathrm{~h}$ after injury. The impact of severity of muscle injury was characterized by the percentage of decrease (\% decrease) in L-BIA $(R, X c$ and PA) compared to the non-injured side.

The adhesive contact electrodes Ag/AgCl (COVIDIEN Ref. 31050522, Covidien LLC, Mansfield, IL, USA) were placed in the area of the injured muscle. The sensing pair of electrodes measuring the voltage $(V)$ drop were placed $5 \mathrm{~cm}$ proximally and $5 \mathrm{~cm}$ distally from the center of the injury, and the source electrodes injecting the current $(I)$ were positioned close to the others. In the vastus intermedius, injuries are located below the rectus femoris and are therefore anatomically deeper, electrodes for detecting $V$ were placed $10 \mathrm{~cm}$ proximally and $10 \mathrm{~cm}$ distally to the center of the injury and two for injecting $I$ close to the others. Similar to previous reports (Nescolarde et al 2013, 2015), the center of the injury was determined by ultrasound (LOGIQ e, GE Healthcare; Milwaukee, WI, USA). For the placement of the electrodes, we had to consider that in deep injuries (near the bone), the current electrodes should be placed with a greater separation than in superficial injuries to increase the depth of the current's penetration. These considerations are in agreement with those reported by Sanchez et al (2016) and more recently by Rutkove et al (2017). The positioning of the electrodes for L-BIA is crucial in correctly assessing muscle injury and following recovery until the RTP because inconsistent electrode positioning can influence the measurement.

Figure 1 shows a localized bioimpedance electrode placement on the injury located in $1 / 3$ medium quadriceps. Points of reference in the center of the injury determined by US, done with a black permanent marker, can be seen on the leg of the football player. The injured players were supine for $15 \mathrm{~min}$ in the medical service $\left(22-24^{\circ} \mathrm{C}\right)$ prior to the L-BIA measurement. 




Figure 1. Tetra-polar localized bioimpedance electrode placement on the injury located in $1 / 3$ medium quadriceps.

The reliability of electrode placement is a critical aspect of L-BIA measurement. The intertester reproducibility for this L-BIA method had a standard deviation (SD) of $0.6 \Omega$ for $R$ and $0.5 \Omega$ for $X c$, with coefficients of variation $(\mathrm{CV})$ of 1.4 and $3.2 \%$, respectively (Nescolarde et al 2013). These estimates are consistent with posterior determinations of repeatability of the L-BIA measurements with a CV of $1.0-1.9 \%$ of the L-BIA measurements except in the adductors, which reached $2.1-3.5 \%$ (Nescolarde et al 2015).

\subsection{Data analysis}

Data are presented as means and standard deviations (SD). The normality of the distribution of the variables was checked by the Shapiro-Wilk test, and the homogeneity of variances by Levene's test.

One-way ANOVA statistical analysis with Scheffé post hoc test or T2-Tamahne (if nonhomogeneity) was used to determine the effect of the severity of injury on relative change (decrease) in $R, X c$ and PA among grades $1,2 \mathrm{f}$ and $2 \mathrm{~g}$, in addition to determining the relationship between the severity of muscle injury and according to grades $1,2 \mathrm{f}$ and $2 \mathrm{~g}$, to days to RTP.

The statistical software IBM® SPSS $®$ version 22.0 (Armonk, NY: IBM Corp, USA) was used for the data analysis. The statistical significance was set at $P<0.05$.

\section{Results}

The 22 muscle injuries were grouped by identification of the gap by MRI exam, either in myotendinous or myofascial areas, in three groups (grade 1 , grade $2 \mathrm{f}$ and grade $2 \mathrm{~g}$ ) and evaluated by L-BIA.

Table 1 shows the L-BIA measurements and percent decreases corresponding to grade 1 $(n=7)$, grade $2 \mathrm{f}(n=8)$ and grade $2 \mathrm{~g}(n=7)$ muscle injuries, significantly in $R(p<0.002)$, $X c(p<0.0001)$ and PA $(p<0.0001)$. The increasing severity of the muscle injury predicted the greater decrease in L-BIA measurements (table 2).

The presence and severity of muscle disruption adversely affected $(p<0.0001)$ time to RTP by 8,14 and $48 \mathrm{~d}$, respectively (figure 2). As shown in table 3 , there was a tendency $(p<0.067)$ for a longer time for RTP between grade 1 (small area of edema) and grade $2 \mathrm{f}$ (moderate amount of edema and no gap) whereas greater differences were found between grade 1 (small area of edema) and grade $2 \mathrm{~g}$ (moderate amount of edema and gap) $(p<0.001)$ 
Table 1. Localized bioelectrical impedance measurements for control (contralateral non-injured side), injured side and percentage decrease in $R, X c$ and PA corresponding to grade 1 , grade $2 \mathrm{f}$ and grade $2 \mathrm{~g}$ injuries.

\begin{tabular}{|c|c|c|c|c|c|c|c|c|c|}
\hline & \multicolumn{3}{|c|}{ Non-injured } & \multicolumn{3}{|c|}{ Injured } & \multicolumn{3}{|c|}{ Decrease $(\%)$} \\
\hline & $R(\Omega)$ & $X c(\Omega)$ & $\mathrm{PA}\left({ }^{\circ}\right)$ & $R(\Omega)$ & $X c(\Omega)$ & $\operatorname{PA}\left({ }^{\circ}\right)$ & $R$ & $X c$ & PA \\
\hline \multirow[t]{2}{*}{ Grade 1} & $37.3^{\mathrm{a}}$ & 14.9 & 22.2 & 33.4 & 12.9 & 21.5 & 10.2 & 13.4 & 3.2 \\
\hline & $7.8^{\mathrm{b}}$ & 1.9 & 3.8 & 6.6 & 1.7 & 3.6 & 2.4 & 1.8 & 2.2 \\
\hline \multirow[t]{2}{*}{ Grade $2 \mathrm{f}$} & 42.1 & 15.1 & 20.1 & 36.7 & 11.6 & 17.8 & 12.8 & 23.5 & 11.2 \\
\hline & 7.8 & 1.8 & 2.9 & 7.0 & 1.4 & 2.4 & 4.9 & 2.2 & 4.5 \\
\hline \multirow[t]{2}{*}{ Grade $2 \mathrm{~g}$} & 41.2 & 16.2 & 22.4 & 32.8 & 10.1 & 17.6 & 19.9 & 37.5 & 20.5 \\
\hline & 13.3 & 2.8 & 4.6 & 10.3 & 1.9 & 3.1 & 5.3 & 7.9 & 6.3 \\
\hline
\end{tabular}

${ }^{\mathrm{a}}$ Mean.

${ }^{\mathrm{b}} \mathrm{SD}$

Table 2. Comparison of percentage of decrease of L-BIA measurements corresponding to grade 1 , grade $2 \mathrm{f}$ and grade $2 \mathrm{~g}$ muscle injuries.

\begin{tabular}{llll}
\hline L-BIA measurement & Comparison & $\mathrm{SE}^{\mathrm{c}}$ & $p$ \\
\hline$R^{\mathrm{b}}$ & 1 versus 2f & 2.35236 & $<0.020$ \\
& $2 \mathrm{f}$ versus 2g & 2.27766 & $<0.021$ \\
\hline \multirow{3}{*}{$c^{\mathrm{c}}$} & 1 versus 2f & 1.02689 & $<0.001$ \\
& 1 versus 2g & 3.04438 & $<0.001$ \\
& $2 \mathrm{f}$ versus 2g & 3.06761 & $<0.001$ \\
\hline \multirow{3}{*}{$\mathrm{PA}^{\mathrm{b}}$} & 1 versus 2f & 2.41639 & $<0.014$ \\
& 1 versus 2g & 2.49564 & $<0.001$ \\
& 2 f versus 2g & 2.41639 & $<0.004$ \\
\hline
\end{tabular}

${ }^{a}$ SE: standard error

${ }^{\mathrm{b}}$ Scheffe.

${ }^{\mathrm{c}}$ Tamahne.

as well as grade $2 \mathrm{f}$ (moderate amount of edema and no gap) and grade $2 \mathrm{~g}$ (moderate amount of edema and gap) muscle injury $(p<0.001)$.

Figures 3-5 show a MRI example of a muscle injury in rectus femoris grade 1 , grade $2 \mathrm{f}$ and grade $2 \mathrm{~g}$, respectively.

\section{Discussion}

Using tetra-polar L-BIA measurements at $50 \mathrm{kHz}$ in lower leg wounds, Lukaski and Moore (2012) found a modest decrease in $R, X c$ and PA acutely after debridement with a considerably greater decrease in the advance of infection, and a substantial increase in $R, X c$ and PA after treatment, and healing is consistent with the hypothesis that L-BIA non-invasively illustrates a cellular level structure previously postulated by the same author (Lukaski 1996). Xc is proportional to cell mass (cell membranes consist of bilayers of lipids and they act as capacitors). Increases in $X c$ indicate epidermal proliferation and granulation whereas $X c$ decreases with infection and cell loss. The R-value is inversely proportional to extra-cellular fluid and directly proportional to the fibrin clot and epithelialization and thus indicates successful wound healing. Healthy membranes cause a delay in the transit of voltage and current; thus, the greater the PA, the healthier the cell membranes, and decreases in PA reflect impaired membrane function. 


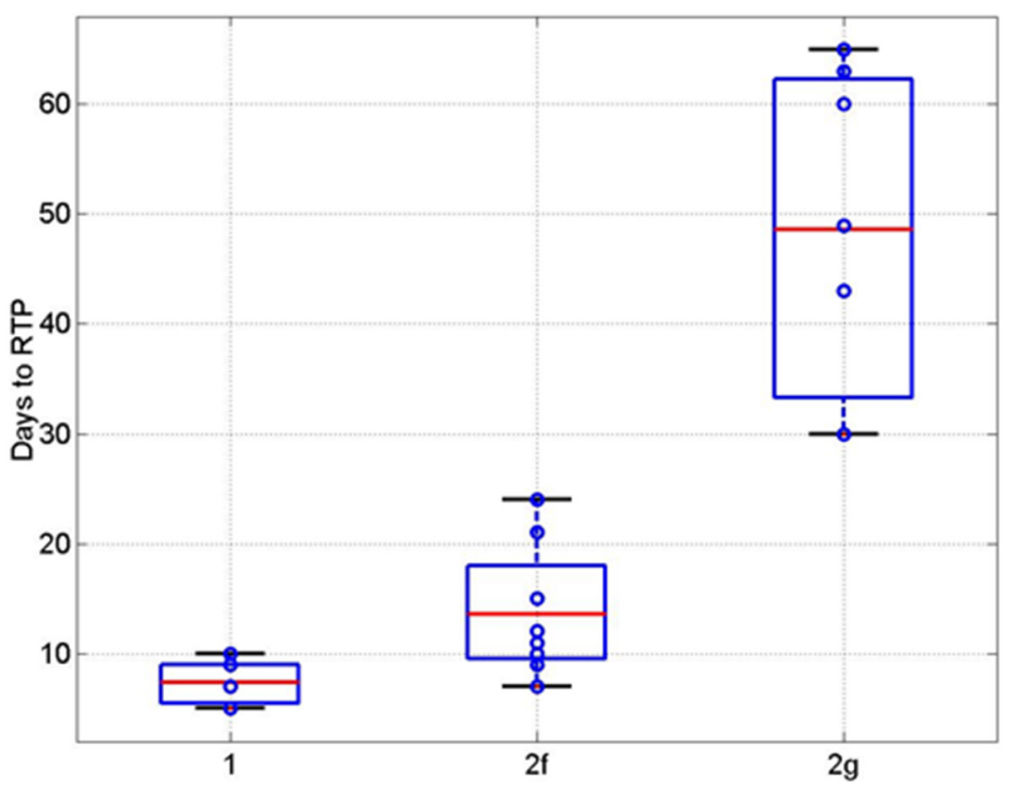

Figure 2. Description of the number of the days from day of injury until day of RTP as box diagrams for injuries grouped by increasing severity of muscle disruption (gap).

Table 3. Results of post hoc analyses of the number of days to RTP for injuries grouped by the presence of muscle disruption (gap).

\begin{tabular}{llll}
\hline Days to RTP & Comparison & SE $^{\mathrm{a}}$ & $p$ \\
\hline \multirow{3}{*}{ T2-Tamahne } & 1 versus 2f & 2.24998 & 0.067 \\
& 1 versus 2g & 5.67666 & 0.001 \\
2f versus 2g & 6.01312 & 0.001 \\
\hline
\end{tabular}

${ }^{\mathrm{a}} \mathrm{SE}$ : standard error.

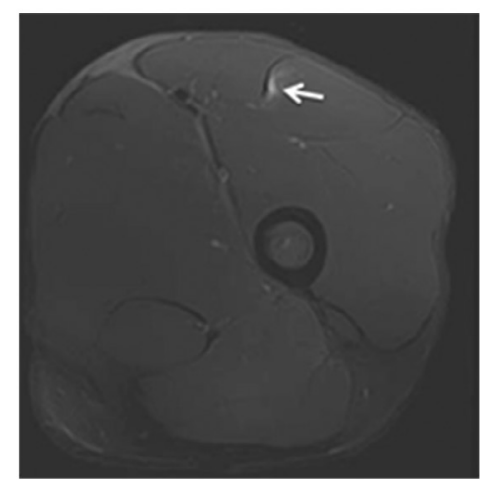

(a)

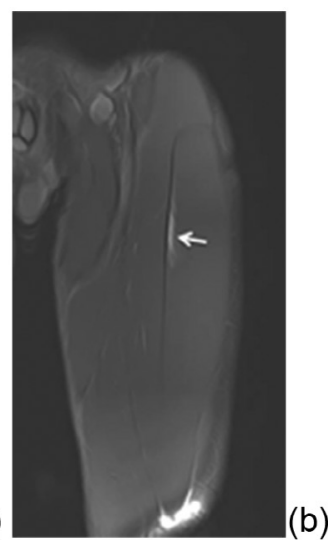

Figure 3. (Grade 1) Fat-suppressed T2-weighted axial (a) and coronal (b) image showing a minimal area of edema (arrows) involving the myotendinous junction with no gap. 

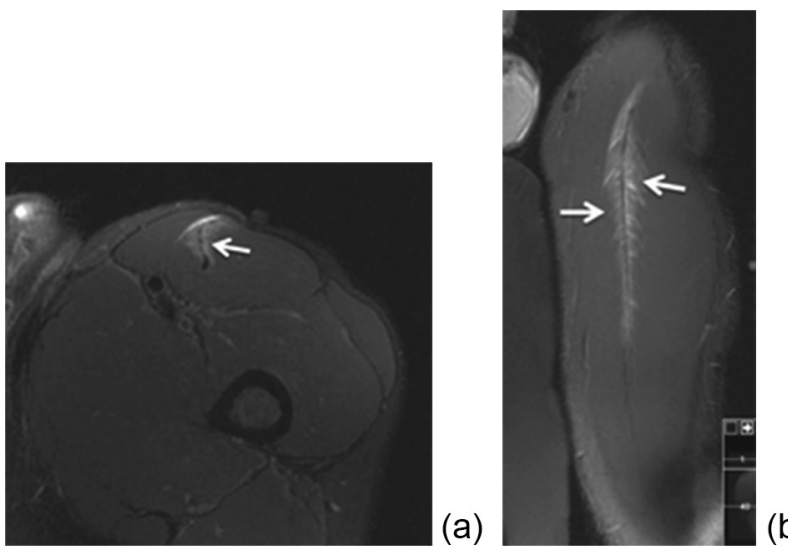

Figure 4. (Grade 2f) Fat-suppressed T2-weighted MRI axial (a) and coronal (b) images showing a moderate feather-like edema (arrows) affecting the myotendinous junction with no gap.
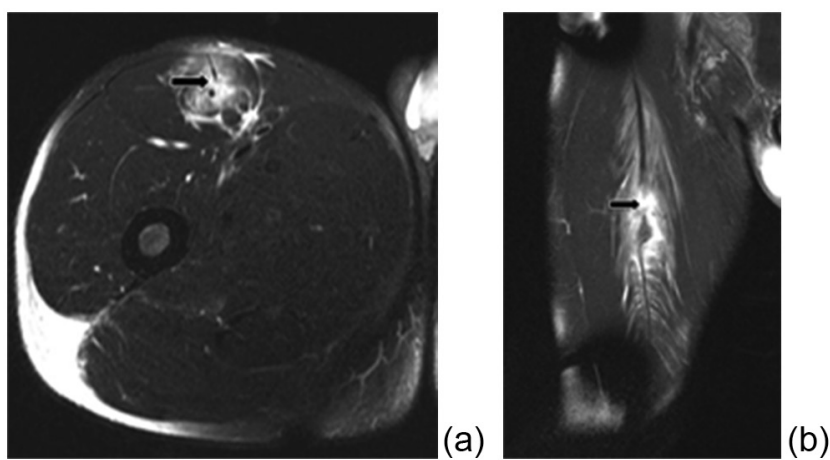

Figure 5. (Grade 2g) Fat-suppressed T2-weighted MRI axial (a) and coronal (b) images showing a moderate feather-like hyper-intense edema affecting the myotendinous junction with a clear gap (thick black arrows) showing loss of integrity of the muscletendon complex.

Later Nescolarde et al $(2013,2015)$, found a similar pattern to the aforementioned study in muscle injuries in professional football players, reporting quantitatively a pattern of change in $R$ and $X c$ according to the severity of the injury in terms of 'grade I' (minor injury), 'grade II' (partial tear or moderate injury) and 'grade III' (complete rupture or severe injury). The most significant changes were observed in severe injuries, showing statistical significance in changes in $X c$ (muscle fibers retraction, that is 'gap') but not in $R$ (edema). Thus, the authors concluded that, according to previous studies based on MRI criteria (Schneider-Kolsky et al 2006, Reurink et al 2015), the presence of edema in the injured muscle did not correlate with the grade of the injury.

This work presents 22 muscle injuries in 18 football players. Table 1 describes the value of the contralateral, non-injured side, $24 \mathrm{~h}$ after injury and the percentage of decrease in $R, X c$ and PA according to the presence of edema and/or gap by MRI. The results of the one-way ANOVA test of percentage of decrease for seven muscle injuries classified as grade 1,8 as grade $2 \mathrm{f}$ and seven injuries as grade $2 \mathrm{~g}$ shows high statistical significance among grades 1 , 2f and $2 \mathrm{~g}$ for $R(F=9.126, p<0.002), X c(F=46.269, p<0.000)$ and PA $(F=24.164$, 
$p<0.000$ ). Due to the low number of grade III injuries (complete muscle ruptures involving in addition the tendon) in football we have not used them for this work.

Recently, a new classification of muscle injuries was proposed (Valle et al 2016) where the gap plays an important role, with the drawback that it is not always possible to identify by MRI. That is the reason why we have divided the classical 'partial muscle tears' (grade II, partial ruptures or moderate injuries) into subgroups: $2 \mathrm{f}$ (feather-like MRI with no gap) and $2 \mathrm{~g}$ (featherlike MRI and gap image). Both are partial tears but the MRI could not detect small gaps in some of them and the classification, based on MRI, will be determined by a small amount of edema (grade I) or much more extension (grade II). The prognosis of the injury and the RTP time will depend on, among others factors, on the severity of the injury and this will be incorrect if the classification of the injury is mistaken. The post hoc analysis shown in table 2 reveals a high statistical difference in $X c$ and PA contrasting grade 1 with grade $2 \mathrm{f}$, grade 1 with grade $2 \mathrm{~g}$ and grade $2 \mathrm{f}$ with grade $2 \mathrm{~g}$. Only in the case of where no statistical differences were observed contrasting grade 1 with grade $2 \mathrm{f}$ with the $R$ parameter. So it is interesting to better differentiate the great and heterogeneous group of partial tears in two subgroups because significant changes in $X c$ could help us give real importance to muscle injury demands and therefore provide the best possible prognosis. Without a doubt, new future MRI devices will be able to detect the very little gaps in small grade II injuries that L-BIA is starting to reveal.

Regarding RTP, grade 1 ( $7 \pm 2 \mathrm{~d}), 2 f(14 \pm 6 \mathrm{~d})$ and $2 g(48 \pm 15 \mathrm{~d})$, a high statistical difference using one-way ANOVA test $(F=41.286, p<0.000)$ was also found. The results of the T2-Tamahne post hoc test show a high statistical difference contrasting grade 1 with grade $2 \mathrm{~g}$, grade $2 \mathrm{f}$ with grade $2 \mathrm{~g}$ and a slight statistical difference between grade 1 and grade $2 \mathrm{f}$. These results are shown in table 3 and figure 2. A slight statistical relevance between grade 1 and grade $2 \mathrm{f}$ injuries leads us to think that the gap that could exist in grade $2 \mathrm{f}$ (not detectable nowadays by MRI) is so small that the recovery time until the RTP could be similar. The great difference is between grades $2 \mathrm{f}$ and $2 \mathrm{~g}$, nowadays considered as injuries belonging to the same group but with very different prognoses between them.

\section{Conclusion}

We conclude that the grade of the muscle injury is proportional to the size of the gap, and these results reveal that gap and the severity of the muscle disruption adversely affect RTP time. L-BIA could help in arriving at a diagnosis and in establishing a prognosis for the RTP time.

\section{Acknowledgment}

The authors especially wish to thank the players of Futbol Club Barcelona and Jordi Urpí Bellós for their collaboration in this study.

\section{References}

Connell D A et al 2014 Longitudinal study comparing sonographic and MRI assessments of acute and healing hamstring injuries Am. J. Roentgenol. $183975-84$

Ekstrand J, Healy J C, Waldén M, Lee J C, English B and Hägglund M 2012 Hamstring muscle injuries in professional football: the correlation of MRI findings with return to play Br. J. Sports. Med. $46112-7$ 
Hamilton B, Valle X, Rodas G, Til L, Pruna Grive R, Gutierrez Rincon J A and Tol J L 2014 Classification and grading of muscle injuries: a narrative review Br. J. Sports. Med. 49306

Lukaski H C 1996 Biological indexes considered in the derivation of the bioelectrical impedance analysis Am. J. Clin. Nutr. 64 391S-404S

Lukaski H C 2013 Evolution of bioimpedance: a circuitous journey from estimation of physiological function to assessment of body composition and a return to clinical research Eur. J. Clin. Nutr. 67 2S-9S

Lukaski H C and Moore M 2012 Bioelectrical impedance assessment of wound healing J. Diabetes Sci. Technol. 6 209-12

Nescolarde L, Yanguas J, Lukaski H, Alomar X, Rosell-Ferrer J and Rodas G 2013 Localized bioimpedance to assess muscle injury Physiol. Meas. 34 237-45

Nescolarde L, Yanguas J, Lukaski H, Alomar X, Rosell-Ferrer J and Rodas G 2015 Effects of muscle injury severity on localized bioimpedance measurements Physiol. Meas. 36 27-42

Pedret C, Rodas G, Balius R, Capdevila L L, Bossy M, Vernooij R W M and Alomar X 2015 Return to play after soleus muscle injuries Orthop. J. Sports Med. 3

Reurink G, Brilman E G, de Vos R J, Maas M, Moen M H, Weir A, Goudswaard G J and Tol J L 2015 Magnetic resonance imaging in acute hamstring injury: can we provide a return to play prognosis? Sports Med. $45133-46$

Rutkove S, Pacheck A and Sanchez B 2017 Sensitivity distribution simulations of surface electrode configurations for electrical impedance myography Muscle Nerve (https://doi.org/10.1002/ mus.25561)

Sanchez B, Pacheck A and Rutkove S B 2016 Guidelines to electrode positioning for human and animal electrical impedance myography research Sci. Rep. 632615

Schneider-Kolsky M E et al 2006 A comparison between clinical assessment and magnetic resonance imaging of acute hamstring injuries Am. J. Sports Med. 34 1008-15

Slavotinek J P, Verrall G M and Fon G T 2002 Hamstring injury in athletes: using MR imaging measurements to compare extent of muscle injury with amount of time lost from competition Am. J. Roentgenol. 179 1621-8

Valle X et al 2016 Muscle injuries in sports: a new evidence-informed and expert consensus-based classification with clinical application Sports Med.

Verrall G M, Slavotinek J P, Barnes P G, Fon G T and Esterman A 2006 Assessment of physical examination and magnetic resonance imaging findings of hamstring injury as predictors for recurrent injury J. Orthop. Sports Phys. Ther. 36 215-24 